\title{
SEVENTEEN OBSTACLES \\ FOR RESOLUTION OF SINGULARITIES
}

\author{
Herwig Hauser
}

This is the first of a series of papers related to resolution of singularities. We present here examples which explain why many arguments and proofs work in special situations, say small dimension or zero characteristic, but fail in general. This exhibits in particular the delicacy of resolution of singularity for arbitrary excellent schemes. The examples were originally assembled for the author's personal records. They might be of some interest to a larger audience, especially to readers for whom the flavour of resolution of singularities is concealed by technique.

We shall concentrate here on the classical approach developed by Zariski, Abhyankar, Hironaka and several other mathematicians towards an inductive proof of resolution of singularities by a sequence of well chosen monoidal transformations [A 1, H 1, BM 1, Sp 1, V 1, Z 1]. The basic idea is to construct sufficiently fine local invariants of singularities which determine the center of blowing up at each stage as the locus of points on the variety where the invariants take their maximal values and to measure the improvement the variety undergoes when passing to the blown up variety by comparing these and possibly further invariants before and after the blowup.

At present there is no completely satisfying answer to this objective. One reason is the lack of conceptuality of the proposed and studied invariants, already apparent in characteristic 0 , the other, which is partly a consequence of the first, that the known invariants sometimes behave badly under blowup in positive characteristic.

We shall set up a catalogue of cautions one has to be aware of when using standard resolution invariants or searching new ones. In principle, there are two types of local arguments involved: a combinatorial one, which investigates resolution with respect to fixed coordinate systems and only treats the origins of the charts in the blown up varieties, and a reduction argument, which intends to reduce the general situation where neither coordinates nor the point of the exceptional divisor are specified to the combinatorial situation.

The combinatorial problem is also known as Hironaka's polyhedral game. In the context of valuations it has been solved by Zariski [Z 2, p. 861]. The solution proposed by Spivakovsky [Sp 2] relies on invariants already considered by Hironaka. However, these invariants cause difficulties for 
the reduction problem, especially in positive characteristic, which have not been overcome yet, see examples 16 and 17 . We meet here a typical phenomenon of resolution of singularities: the various detail problems have to be solved coherently and simultaneously in the sense that each separate solution to one problem only serves if it has been suitably adjusted with respect to the solutions of the other problems.

Our exposition is confined to the local problem, i.e. the definition of local invariants of singularities and their behaviour under blowing up. This is the core of the problem, though global aspects are not to be neglected. We shall deprive our presentation from technical and sophisticated decoration. The main obstructions already appear in simple and elementary circumstances.

Recently, there has opened an entirely new approach to resolution of singularities through work of de Jong. His method combines induction based on semistable reduction with arguments from toric geometry and proves resolution of singularities up to a finite map in any characteristic $[\mathrm{J}]$. See Berthelot's Bourbaki note [Be] for another account on this as well as various applications. There are subsequent papers of Abramovich, de Jong, respectively, Bogomolov and Pantev extending these techniques and proving on a few pages a weak version of Hironaka's theorem in characteristic zero [AJ, BP]. The difference to Hironaka's version is that centers are blown up which are not necessarily contained in the singular locus of the variety and hence the variety is also modified in regular points.

Let us formulate the strong version of resolution of singularities in the context of varieties over a field (i.e. schemes of finite type over a field):

Embedded Resolution of Singularities. Given a reduced variety $X$ over a field $\mathbf{K}$ and embedded in a smooth variety $W$, construct a smooth variety $\tilde{W}$ and a proper birational morphism $\pi: \tilde{W} \rightarrow W$ such that the inverse image $\tilde{X}$ of $X$ under $\pi$ has only normal crossing singularities and such that the restriction of $\pi$ over the smooth points of $X$ is an isomorphism.

Recall that a variety is said to have normal crossing singularities if it consists of a union of smooth components intersecting transversally, more precisely, such that at any intersection point there is a coordinate system for which the variety is defined locally by monomials. The most explicit way to construct $\tilde{W}$ is by a finite sequence of well chosen blowups. This requires to determine the centers of each blowup as a subvariety of the singular locus Sing $X$ of $X$ and to show that after a finite number of steps only normal crossing singularities are left. It is convenient, even though 
a priori more difficult, to treat the embedded situation. This allows to work with regular systems of parameters which are very helpful to define local invariants. Then to prove embedded resolution of singularities of varieties it suffices to solve the following problem:

Inductive Resolution Problem. Given a field $\mathbf{K}$ and a smooth variety $W$ over $\mathbf{K}$, construct the following objects:

(1) Centers: A map

$Z:\{$ reduced subvarieties $X$ of $W\} \rightarrow\{$ subvarieties of $\operatorname{Sing} X\}$

such that the blowup $\pi: \tilde{W} \rightarrow W$ of $W$ in $Z_{X}$ gives a smooth variety $\tilde{W}$.

(2) Resolution invariant: A well-ordered set $\Gamma$ and a map

$i:\{$ reduced subvarieties $X$ of $W$ with a point $p$ on $X\} \rightarrow \Gamma$

such that for $\tilde{X}=\pi^{-1} X$ the following conditions are satisfied:

(i) $i_{p} X=0$ if and only if $X$ has normal crossing at $p$.

(ii) $i_{p^{\prime}} \tilde{X}<i_{p} X$ for all $p \in Z_{X}$ and $p^{\prime} \in D=\pi^{-1} Z_{X}$.

(iii) $i$ is upper semicontinuous w.r.t. $p$.

(iv) $\left\{p \in X, \quad i_{p} X\right.$ maximal on $\left.X\right\} \subset Z_{X}$.

As a variant, replace (i) by $i_{p} X=0$ if and only if $X$ is smooth at $p$ and in (ii) the total transform $\tilde{X}$ by the strict transform $X^{\prime}$, cf. section B. Observe that in contrast to the classical notion of permissible centers as in [H 1, III.5] we allow singular and even non-reduced centers $Z_{X}$, provided $\tilde{W}$ is smooth, see section B. and the discussion after example 3. Some authors require equality in (iv), i.e., that $Z_{X}$ is the smallest stratum of the stratification of $X$ induced by $i$ [BM 1].

The resolution invariant $i$ is usually a vector of integer or rational invariants equipped with the lexicographic order. Typically it is shown that its first component does not increase under blowup. If it remains constant, the situation must be sufficiently specific to be able to show that the second component does not increase. Then the argument is repeated, until one arrives at a component which strictly decreases.

The definition of the centers is a global problem, whereas the invariants are of local nature. However, often local invariants are used to define the centers locally, and then patching arguments are needed to show that the local constructions expand to give global centers [V 1, V 2]. Some authors restrict even further the nature of the invariants as to be defined along a valuation, cf. with the concept of uniformization $[\mathrm{Z} 2, \mathrm{Sp} 1]$. It 
then suffices to prove that the invariant has dropped only at the point of the exceptional divisor which is determined by the valuation.

The four conditions above allow to apply the following induction argument: The stratification of $X$ by subvarieties along which $i$ is constant is locally finite by (iii) and since $X$ is Noetherian. By (ii) and (iv) each point of the worst stratum $\left\{p \in X, i_{p} X\right.$ maximal on $\left.X\right\}$ improves under the blowup. Hence the maximal value of $i$ on $X$ drops when passing to $X^{\prime}$. As $\Gamma$ is well ordered, it becomes 0 in a finite number of steps, and (i) applies.

Let us comment on the induction invariants usually considered. The most important one is the order of the defining ideal, i.e. the largest power of the maximal ideal which contains the ideal. For hypersurfaces it is just the order of the series expansion of $f$ w.r.t. some coordinate system. By a theorem of Nagata-Zariski the order does not increase under localization, i.e. is upper semicontinuous [H 1, p. 218]. Also it is known not to increase under permissible blowup in any dimension and characteristic (infinitesimal upper semicontinuity.) The concept of multiplicity has been extended by Hironaka to the non hypersurface case by introducing the finite sequence $\nu^{*}$ of integers given as the orders of the elements of a standard basis of the defining ideal, listed increasingly and ordered lexicographically, cf. [H 1, III.1] and section E.(5) below. It does not increase under permissible blowing up [H 1, III. Thm. 3], but may behave badly under localization, see example 11. Bennett suggested to replace $\nu^{*}$ by the Hilbert-Samuel function of the local ring and established its infinitesimal and ordinary semicontinuity for arbitrary excellent schemes [Bn, Si]. It is generally accepted today (though not the conviction of the author) that the Hilbert-Samuel function should be the first invariant to be considered, at least for the definition of the centers.

The next step is to investigate the situation where, say, the HilbertSamuel function remains constant under blowup. Further invariants are then necessary. There are several options appearing in the literature, depending on the context. Hironaka and Abhyankar propose, already in the hypersurface case, to take the minimal number of variables necessary to define the initial form of $f$, cf. section E.(2) and example 7. Other invariants can be constructed from the Newton polyhedron associated to the power series expansion of $f$. These are often generalizations of the notion of maximal slope for plane curves, sometimes known as weighted orders.

A very intricate problem consists in measuring the singularities of the equimultiple locus. For technical reasons we have to be vague here and must refer the reader to [H 5, BM 2, V 1] for more details. Simplifying 
first the singularities of the equimultiple locus is crucial in order to be able to define sufficiently big centers for resolving the variety itself, cf. example 1. This requires to apply induction on invariants related to the equimultiple locus. Therefore the local invariants associated to $X$ will also involve invariants of 'smaller' varieties. In characteristic 0 , a precise meaning can be given to the comparative 'smaller' through the local embedding dimension and the theory of idealistic presentations, but this fails in positive characteristic and still remains unsettled, cf. example 8 . We know of no appropriate method in characteristic $p>0$ to handle this difficulty in arbitrary dimension. See [A 2] for an explicit description of the problems in the curve case.

Most of the examples below illustrate situations where certain of these invariants do not to satisfy (ii) or (iii) in general circumstances. Other examples point at problems which occur in the definition of the centers and show why the construction of new invariants is very subtle and delicate. In [Ha 1, Ha 2, Ha 3, Ha 6] we develop techniques which allow the systematic construction of invariants and provide a uniform frame for many of the known invariants. This seems to be a prerequisite towards a conceptual approach to the inductive resolution problem. Up to now, many invariants and resolution arguments are established ad hoc by defining all kinds of distinguished coordinate systems and introducing some numerical data associated to them. You may find coordinates which are prepared, well-prepared, very well-prepared [H 3], tangential, normal [H 6], good, essential, nontangential [A 4], essential [BM 1], good, quasi-good [LO], maximal clean, Zariski clean [Mo 1, Mo 2], convenient [V 2], just to list a few of the used labels. All these notions follow the same pattern. Namely, they can be characterized as coordinates which maximize some numerical datum attached to the singularity [Ha 1].

We do not discuss in this article methods and topics as normalization, Nash modification, toroidal resolution, uniformization, rectilinearization, valuation theory, resolution of surfaces or quasi-ordinary singularities, see for instance [Br 1, Br 2, GT, KKMS, La, Lp 2, Ok, OW, Su, Z 2, Z 5, Z 6]. For further introductions and surveys we refer to [A 2, A 3, A 4, Ar, Bd, BM 3, CGO, Co 3, Gi 1, Gr 1, H 6, H 7, Lp 1, Od 1, Od 3, SG $1, \mathrm{~V} 3]$. References for some historical papers are [Al, Ch, Ju, Lv $1, \mathrm{Lv}$ $2, \mathrm{~N}, \mathrm{NB}, \mathrm{W}]$. We apologize for any omission or incompleteness which might have occurred in citing results and techniques from the literature.

The preparation of this article was supported by a grants from the Austrian-Spanish cooperation program Acciones Integradas and the D. Swarovski Forschungsförderungsfonds. We thank O. Villamayor, V. Cossart and M. Spivakovsky for valuable suggestions and references. 


\section{B. Basics}

We start by recalling some fundamental notions on blowups. References are e.g. [H 1, III.2, H 3, H 6, Ha 5, Hs, II.7, Gi 1, HIO, Bb, Gr 2, IV.7, $\mathrm{K}]$. Let us place in some $n$-dimensional affine space $\mathbf{A}^{n}$ over a field $\mathbf{K}$ and fix a point $p$ in $\mathbf{A}^{n}$. Since we are interested in local questions at $p$, we accordingly fix a regular local Noetherian ring $\mathbf{R}$ of dimension $n$ and maximal ideal $\mathbf{M}$. Without to much loose of generality we suppose that $\mathbf{R}$ is complete. Moreover we shall assume $\mathbf{R}$ equicharacteristic, so that, by Cohen's Structure Theorem, $\mathbf{R}$ can be viewn as a formal power series ring in $n$ variables over $\mathbf{K}$. With the exception of example 7 , the reader may think of $\mathbf{K}$ as being algebraically closed.

Any non-zero ideal $\mathbf{P}$ of $\mathbf{R}$ gives rise to the Rees algebra or blowup algebra $\mathbf{S}=\oplus_{i \geq 0} \mathbf{P}^{i}$ of $\mathbf{R}$ with center $\mathbf{P}$. Here, $\mathbf{P}^{0}=\mathbf{R}$, so that $\mathbf{R} \subset \mathbf{S}$. Localizing $\mathbf{S}$ at a maximal ideal $\mathbf{Q}^{\prime}$ which contains $\mathbf{M}$ and passing to the completion gives a complete local ring $\mathbf{R}^{\prime}$ and an inclusion $\pi^{*}: \mathbf{R} \rightarrow \mathbf{R}^{\prime}$ which we shall call the blowup of $\mathbf{R}$ with center $\mathbf{P}$ considered locally at $\mathbf{Q}^{\prime}$. Geometrically, this corresponds to blow up $\mathbf{A}^{n}$ in the subvariety $Z$ defined by $\mathbf{P}$ and to look at $\tilde{\mathbf{A}}^{n}=\operatorname{Proj} \mathbf{S}$ locally at the point $p^{\prime}$ defined by $\mathbf{Q}^{\prime}$ in the exceptional divisor $D=\pi^{-1} Z$, where $\pi: \tilde{\mathbf{A}}^{n} \rightarrow \mathbf{A}^{n}$ denotes the projection associated to $\mathbf{R} \subset \mathbf{S}$.

Any set of generators $a_{1}, \ldots, a_{k}$ of $\mathbf{P}$ induces a covering of $\tilde{\mathbf{A}}^{n}$ by affine charts Proj $\mathbf{S}=\bigcup_{i=1}^{k} \operatorname{Spec} \mathbf{R}\left[\frac{\mathbf{P}}{a_{i}}\right]=\bigcup_{i=1}^{k} \operatorname{Spec} \mathbf{R}\left[\frac{a_{1}}{a_{i}}, \ldots, \frac{a_{k}}{a_{i}}\right]$. Each chart is specified by selecting one generator $a_{j}$. Letting $\mathbf{Q}$ be the ideal of $\mathbf{R}$ generated by the remaining ones we have $\mathbf{R}\left[\frac{\mathbf{P}}{a_{j}}\right]=\mathbf{R}\left[\frac{\mathbf{Q}}{a_{j}}\right]$. In particular, $\mathbf{Q}$, or $j$, determine a point $p^{\prime}$ in $D$, namely the origin of this affine chart. More generally, any affine chart on $\tilde{\mathbf{A}}^{n}$ can be given by selecting an ideal $\mathbf{Q} \subset \mathbf{P}$ of $\mathbf{R}$ with $\mathbf{P} / \mathbf{Q}$ of height 1 in $\mathbf{R} / \mathbf{Q}$. Take any element $a \in \mathbf{P}$ whose class generates $\mathbf{P} / \mathbf{Q}$ and get the chart $\mathbf{R}\left[\frac{\mathbf{Q}}{a}\right]$. It turns out that changing $\mathbf{Q}$ by elements from $\mathbf{P}^{2}$ does not alter the chart, i.e. that the chart is determined by the image of $\mathbf{Q}$ in $\mathbf{P} / \mathbf{P}^{2}$. We shall always specify the point $p^{\prime}$ of the exceptional divisor or the chart we are looking at by giving the ideal $\mathbf{Q}$ of $\mathbf{R}$. Geometrically, this is reflected by the fact that any point of the exceptional divisor corresponds to a direction in the normal cone of $Z$ in $\mathbf{A}^{n}$.

If $x_{1}, \ldots, x_{n}$ are local coordinates of $\mathbf{A}^{n}$ at $p$, i.e., a regular system of parameters of $\mathbf{R}$, then $\mathbf{R} \cong \mathbf{K}[[x]]$ and the $j$-th affine chart $\mathbf{K}[[x]]\left[\frac{\mathbf{P}}{a_{j}}\right]$ gives rise to an explicit description when $\mathbf{P}$ is generated by part of the coordinates, say $x_{i}$ with $i \in J$, where $J \subset\{1, \ldots, n\}$ and $j \in J$. Up to permutation, it suffices to consider $J=\{1, \ldots, j\}$ for some $j$, say $\mathbf{P}=\left(x_{1}, \ldots, x_{j}\right)$ and $\mathbf{Q}=\left(x_{1}, \ldots, x_{j-1}\right)$. In this way $\mathbf{K}[[x]]\left[\frac{\mathbf{P}}{a_{j}}\right]$ 
equals $\mathbf{K}[[x]]\left[\frac{x_{1}}{x_{j}}, \ldots, \frac{x_{j-1}}{x_{j}}\right]$. Localization and completion at the ideal $\left(\frac{x_{1}}{x_{j}}, \ldots, \frac{x_{j-1}}{x_{j}}, x_{j}, \ldots, x_{n}\right)$ gives $\mathbf{R}^{\prime}=\mathbf{K}\left[\left[\frac{x_{1}}{x_{j}}, \ldots, \frac{x_{j-1}}{x_{j}}, x_{j}, \ldots, x_{n}\right]\right]$, which is again a regular complete Noetherian local ring of dimension $n$. Hence setting $y_{i}=\frac{x_{i}}{x_{i}}$ for $i<j$ and $y_{i}=x_{i}$ else defines a regular system of parameters for $\mathbf{R}^{\prime}$. The map $\pi^{*}: \mathbf{R} \rightarrow \mathbf{R}^{\prime}$ is then given by $x_{i} \rightarrow y_{i} y_{j}$ for $i<j$ and $x_{i} \rightarrow y_{i}$ for $i \geq j$.

In this situation we say that the local blowing up is monomial of type $j \in J$ w.r.t. the coordinates $x$. The coordinates $y$ in $\mathbf{R}^{\prime}$ are called the induced coordinates, often denoted again by $x$ for convenience. The exceptional divisor $D$ is defined by the principal ideal $\left(y_{j}\right)$.

Observe that the map $\pi^{*}$ is still very simple when $\mathbf{P}$ is generated by monomials in the variables, though $\mathbf{R}^{\prime}$ may then fail to be regular, cf. example 3. Compare this with toric modifications and toric singularities [GT, KKMS, Ok].

Consider now a variety $X$ in $\mathbf{A}^{n}$. Let $\mathbf{I} \subset \mathbf{R}$ be the ideal defining $X$ locally at $p$. Its total transform $\tilde{X}$ is the inverse image of $X$ under $\pi$ : $\tilde{\mathbf{A}}^{n} \rightarrow \mathbf{A}^{n}$. In the $j$-th affine chart it is defined by the ideal $\tilde{\mathbf{I}}=\left(\pi^{*} \mathbf{I}\right) \mathbf{R}^{\prime}$ of $\mathbf{R}^{\prime}$. We shall write $\tilde{\mathbf{I}}=\mathbf{I} \mathbf{R}^{\prime}$ for short. The strict transform $X^{\prime}$ of $X$ is the closure in $\tilde{\mathbf{A}}^{n}$ of the inverse image of $X \backslash Z$ under $\pi$. Its ideal is $\mathbf{I}^{\prime}=\bigcup_{m \geq 0} y_{j}^{-m}\left(\mathbf{I} \cap \mathbf{P}^{m}\right) \mathbf{R}^{\prime}$. In particular, for hypersurfaces $f$, the strict transform equals $f^{\prime}=y_{j}^{-o} f$ where $o$ denotes the order of $f$ w.r.t. $\mathbf{P}$, $o=\max \left\{m, f \in \mathbf{P}^{m}\right\}$. For arbitrary ideals, the strict transform can be computed in terms of generators of the ideal by means of standard bases [H 1, III.2].

The center $\mathbf{P}$ is permissible for a hypersurface $X$ if the order of $f$ w.r.t. $\mathbf{P}$ and $\mathbf{M}$ is the same, i.e., if $Z$ is contained in the equimultiple locus of $X$. In general, $\mathbf{P}$ is permissible if the Hilbert-Samuel function of $X$ is constant along $Z$ [Bn, Thm. 3]. In the literature, $Z$ is assumed smooth, but we do not impose this restriction here.

Assume that we are in the monomial situation of type $j \in J$ w.r.t. coordinates $x$ in $\mathbf{R}$, i.e. $\mathbf{P}=\left(x_{1}, \ldots, x_{j}\right)$ and $\mathbf{Q}=\left(x_{1}, \ldots, x_{j-1}\right)$. Let $y$ be the induced coordinates in $\mathbf{R}^{\prime}$. Denote by $f=\sum c_{\alpha} x^{\alpha}$ the expansion of $f$ w.r.t. $x$ and let $f^{\prime}$ be its strict transform. Since $\pi^{*}$ is given by substitution of the variables by monomials, $f^{\prime}$ has expansion $f^{\prime}=\sum c_{\alpha} y^{\alpha^{*}}$ where the map ${ }^{*}: \operatorname{supp} f \rightarrow \operatorname{supp} f^{\prime}$ is defined by the following formula:

$$
\begin{aligned}
& \alpha_{i}^{*}=\alpha_{i} \quad \text { for } i \neq j, \\
& \alpha_{j}^{*}=\alpha_{j}+\left(\alpha_{1}+\ldots+\alpha_{j-1}-o\right) .
\end{aligned}
$$

Here supp $f=\left\{\alpha \in \mathbf{N}^{n}, c_{\alpha} \neq 0\right\}$ denotes the support of $f$. In the examples below we shall always use this formula to compute the strict 
transform. Let $N_{f}$ denote the Newton polyhedron of $f$, i.e., the convex hull in $\mathrm{R}_{+}^{n}$ of $\operatorname{supp} f+\mathrm{R}_{+}^{n}$. As ${ }^{*}$ is an affine map of $\mathrm{R}^{n}$ the polyhedron $N_{f^{\prime}}$ of the strict transform is computed by taking the convex hull of the images under * of the vertices of $N_{f}$. The improvement of $N_{f^{\prime}}$ relative to $N_{f}$ can be read off in various ways. However, as the Newton polyhedron depends on the chosen coordinates such a measure need not be a local invariant of the singularity. It has to be made coordinate independent, at least to a certain extent. Among the various attempts to extract coordinate free information from the Newton polyhedron, e.g. [CP, H $2, \mathrm{LT}, \mathrm{Y}]$, none suffices to construct an invariant $i$ satisfying (i) to (iv) for arbitrary characteristic. We believe that this is one of the central difficulties in resolution problems. Note that the hypersurface $f=0$ has become a normal crossing singularity at a point of the exceptional divisor if and only if the Newton polyhedron of the total transform has precisely one vertex $\alpha$, and the strict transform of $f$ has become smooth if and only if its Newton polyhedron has a vertex of total degree 1.

\section{E. Examples}

Several of the following examples can be found in the literature or belong to what some people call folklore. The remaining ones have been constructed to clarify the author's view on the subject. In the sequel, $f^{\prime}$ always denotes the strict transform of $f$. The reader is asked to check its asserted form in the examples by computing it in all charts according to the formula for * given above. Occasionally we include exercises and problems. The first are mostly computational and shall consolidate the understanding. For the latter, a complete answer is not always known yet.

(1) Centers of Blowup. For varieties with isolated singularities there is no ambiguity how to choose the center. Only the singular points are permissible. For non-isolated singularities unfortunate choices of the center may yield the same or worse singularities.

Example 1. Let $f=x^{2}+y z$ be the rational double point with isolated singularity at the origin. Blowing up 0 resolves the singularity. For instance, taking the chart corresponding to $\mathbf{Q}=(x, y)$ gives $f^{\prime}=x^{2}+y$ smooth.

Let $f=x^{2}+y^{2} z$ be the Whitney umbrella. The singular locus is the $z$-axis. Since $f$ has order two in each singular point the axis coincides with the equimultiple locus of $f$ (= locus of points of maximal multiplicity.) Two centers are permissible, the origin and the $z$-axis (provided 
that the variety is considered locally at 0.) Exercise: Blowing up 0 reproduces the singularity in the chart $\mathbf{Q}=(x, y)$ whereas blowing up the $z$-axis resolves the singularity. This contradicts our intuition of stratifying the variety according to the complexity of the singularities and to take as center the worst stratum. On first view the origin of the Whitney umbrella seems to be worse than the singularities on the $z$-axis. The example indicates that this naive stratification is too subtle. We have to look for a coarser one. A good recipe is to blow up permissible centers of maximal possible dimension. Such choice is supported by the fact that each singular point has to belong to a center at least once in the resolution process, else it would remain singular until the end. This is an equilibrium problem. Roughly speaking, the centers should be as coarse, the invariants as fine as possible.

Modify the Whitney umbrella to $f=x^{2}+y^{m} z$ with $m \geq 3$. Then for $\mathbf{P}=\mathbf{M}$ and $\mathbf{Q}=(x, y)$ we get $f^{\prime}=x^{2}+y^{m} z^{m-1}$ which is worse than $f$ in whatever sense one may think of. Again we have to blow up the $z$-axis to improve the situation: in the chart $\mathbf{Q}=(y)$ the multiplicity drops, in the chart $\mathbf{Q}=(x)$ we get $f^{\prime}=x^{2}+y^{m-2} z$. Problem: Give a coordinate free description of the improvement of the singularity in the second chart.

Example 2. The Theorem of Beppo Levi asserts that for surfaces whose equimultiple locus has only normal crossing singularities a finite sequence of blowups in smooth permissible centers of maximal dimension makes the multiplicity drop $[\mathrm{Lv} 1, \mathrm{Lv} 2, \mathrm{Z} 1, \mathrm{p} .522]$. The algorithm is not canonical since at certain stages several choices of centers may be possible. The same philosophy fails in higher dimension without further specification of the centers. Spivakovsky gave in [Sp 4] an example for this in dimension 4 , a variant of which is presented here: Let $f=x^{3}+y z^{2} w^{4}+y z^{4} w^{2}$. One checks by inspection that all coordinate axes except the $x$-axis are permissible. Blowing up the $w$-axis, i.e., $\mathbf{P}=(x, y, z)$, the strict transform of $f$ at the point $\mathbf{Q}=(x, z)$ equals $f^{\prime}=x^{3}+z^{2} w^{4}+y^{2} z^{4} w^{2}$. Now the $y$ - and $z$-axis are permissible. Blowing up $\mathbf{P}=(x, y, w)$ gives $f^{\prime \prime}=f$ at the point corresponding to $\mathbf{Q}=(x, w)$. We have run into a cycle of length two.

Observe that each choice of center provides an improvement of the singularity w.r.t. certain variables and a deterioration w.r.t. others. It is then clear that the combination of two blowups may possibly cancel these changes, and that is what happens in the example. A first reaction would be to choose the second blowup according to the first one in order to add the improvements and the deteriorations and then to conclude that if the singularity has sufficiently improved w.r.t. to some variables, 
it has become smooth, regardless the effect w.r.t. the other variables. We know of no effective and intrinsic criterion for this, so let us formulate it as a Problem: measure asymmetric improvements and define the subsequent center depending on the prior blowups and this measure.

A second possible answer is as follows: In the first blowup the situation is symmetric w.r.t. $z$ and $w$. Choosing arbitrarily one of the axes as center destroys this symmetry. The resulting algorithm is no longer canonical. Imagine that the local symmetry of the variety is not induced from a global symmetry. As we consider local invariants to define the center, we will never detect the global asymmetry and it is not reflected in our choice. To avoid this phenomenon we may blow up lower dimensional centers in presence of symmetries. In the example, instead of choosing one axis, we can blow up the origin. This choice is canonical, and keeping track of this preparatory blowing up the local symmetry will disappear along the points of the exceptional divisor, since at an intersection point $p^{\prime}$ of $D$ with the strict transform of the $z$ - or $w$-axis one equimultiple curve, namely the exceptional curve, is new and one, the strict transform of the equimultiple curve from below, is old, hence they can be distinguished. There may still exist a local or even global symmetry of the blown up variety which interchanges the two curves, but our records will tell us that it is not a symmetry of the resolution process. This allows to choose locally at $p^{\prime}$ the new center canonically.

This approach has been applied successfully in the past, cf. [BM 1, V 1]. It burdens enormously the amount of data to define the invariants and notation becomes quite cumbersome. The derived algorithm is usually far from being the most economic one.

Much more effective is to allow in such cases singular centers, with, say, at most normal crossing singularities. The first relevant situation occurs when the equimultiple locus of a surface in $\mathbf{A}^{3}$ consists of two transversal smooth curves as in the example. Classically, singular centers are not treated as permissible. One reason is that blowing up a smooth variety in a singular center generally creates singularities:

Example 3. Blow up two lines in $\mathbf{A}^{3}$, say $\mathbf{P}=(x, y z)$. In the chart $\mathbf{Q}=$ $(y z)$ we have $\mathbf{R}^{\prime}=\mathbf{K}[[x, y, z, x / y z]]=\mathbf{K}[[y, z, x / y z]]$ smooth, whereas for $\mathbf{Q}=(x)$ the ring $\mathbf{R}^{\prime}=\mathbf{K}[[x, y, z, y z / x]] \cong \mathbf{K}[[x, y, z, w]] /(x w-y z)$ is singular.

Pfeifle has determined non reduced structures for normal crossing singularities such that blowing up smooth varieties in the corresponding ideals produces smooth varieties $[\mathrm{P}]$. Since the ideals can be chosen to be monomial, computations are very explicit and can be implemented. 
For the given example, one such non reduced structure on the two lines in $\mathbf{A}^{3}$ is the ideal $\mathbf{P}^{\sharp}=(x, y z)(x, y)(x, z)=\left(x^{3}, x^{2} y, x^{2} z, x y z, y^{2} z^{2}\right)$. It provides five smooth charts for the blown up variety. For instance, in the chart corresponding to $\mathbf{Q}=\left(x^{3}, x^{2} y, x^{2} z, y^{2} z^{2}\right)$ we get

$$
\mathbf{R}^{\prime}=\mathbf{K}\left[\left[x, y, z, \frac{x^{3}}{x y z}, \frac{x^{2} y}{x y z}, \frac{x^{2} z}{x y z}, \frac{y^{2} z^{2}}{x y z}\right]\right]=\mathbf{K}\left[\left[y, \frac{x}{y}, \frac{y z}{x}\right]\right]
$$

which is regular. It is not clear whether there is a general pattern to find these non reduced structures on normal crossing singularities. The problem is related to the resolution of toric singularities (which appear when blowing up a reduced normal crossing ideal), cf. [KKMS]. Exercise: Find another non-reduced structure on two transversal lines in $\mathbf{A}^{3}$ so that the blowup $\tilde{\mathbf{A}}^{3}$ is smooth.

There is a fourth way how to treat multiple possible choices of centers in presence of symmetries. It consists in equipping the variety locally at each of its points with a flag $\mathbf{F}$ of smooth varieties in the ambient space. This corresponds to choosing a maximal chain of regular ideals in $\mathbf{R}$. Assume you blow up a smooth center transversal to all members of the flag. In [Ha 2] it is shown that each point of the exceptional divisor then inherits in a natural way a flag $\mathbf{F}^{\prime}$ induced from the flag below.

This new flag allows to untie symmetries when there appear various candidates as permissible centers by considering their position relative to the flag. Moreover flags allow to define the notion of subordinate coordinate systems. These are coordinates for which the ideals of the defining chain in $\mathbf{R}$ are generated by decreasing collections of them. The set of all subordinate coordinates is clearly an invariant of the pair $(\mathbf{R}, \mathbf{F})$. Using a generalized Gauss-Bruhat decomposition established in [Ha 2] for the automorphism group Aut $\mathbf{R}$ of $\mathbf{R}$ it is possible to show that a whole sector of the Newton polyhedron of a hypersurface $f$ is fixed under automorphisms of $\mathbf{R}$ which preserve subordinate coordinates. Therefore, this portion of vertices is also an invariant of the flagged ambient space $\mathbf{R}$. Now it turns out that subordinate coordinates remain subordinate under blowing up, that is, w.r.t. the induced flag in $\mathbf{R}^{\prime}$. It follows that the invariant part of the Newton polyhedron corresponding to this sector can be compared explicitly before and after the blowup. This is relevant for constructing components of a suitable resolution invariant $i$.

(2) Multiplicity. For $f \in \mathbf{R}$ let $o$ respectively $o^{\prime}$ denote the multiplicity of $f$ and of its strict transform $f^{\prime}$. As mentioned above, $o^{\prime} \leq o$ for any permissible blowing up. In general, equality may hold:

Example 4. $f=x^{2}+y^{5}, \mathbf{P}=(x, y), \mathbf{Q}=(x)$, then $f^{\prime}=x^{2}+y^{3}$ has again multiplicity 2. In order to measure the improvement we are led 
to consider the exponent of the $y$-monomial. This is not intrinsic since there might be several monomials in the expansion of $f$ to look at, even if we assume $f$ given in Weierstrass form. Besides, the expansion depends on the coordinates. For plane curves as in the example, the classical second invariant after the multiplicity is the slope of the Newton polygon of $f$. Assume $f$ is given in Weierstrass form $f=x^{o}+\sum_{i<o} c_{i} x^{i}$ with series $c_{i}$ in $y$. Then the slope of $f$ is defined as the slope of the first segment of the Newton polygon of $f$, i.e. ord $\left(c_{i}\right) /(i-o)$ where $i$ is the largest index for which $c_{i}$ does not vanish identically. In the example the slope passes from $-2 / 5$ to $-2 / 3$. If, in general, it becomes $<-1$, the multiplicity has dropped. If the slope arrives precisely at -1 the multiplicity drops in the next blow up. This reasoning is not exact since the slope as defined above may vary under changes of coordinates. To make it coordinate independent, take the maximal slope which occurs for all coordinate systems. Then the same assertions hold and form the basis for inductive resolution of plane curve singularities, see [BK] for an extensive treatment, and [A 2] for the case of positive characteristic. The notion of maximality is discussed in section E.(6). There is another constructive way to resolve plane curve singularities via toric modifications defined through the fan associated to the Newton polygon [GT, Ok]. Problem: Prove inductively resolution of plane curve singularities by point blowups without using the multiplicity as induction invariant. Such proof becomes necessary when reducing the resolution of surfaces to the plane curve case, see [H 3] and [Ha 3] for a discussion and an answer. Blowups for which the multiplicity remains constant are characterized in $[\mathrm{SG} 2]$.

Example 5. Blow up the plane curve $f=x^{2}-y^{2}+y^{5}$ in the origin. The strict transform intersects the exceptional divisor $D$ in $\tilde{\mathbf{A}}^{2}$ in two points corresponding to $\mathbf{Q}_{1}=(x+y)$ and $\mathbf{Q}_{2}=(x-y)$. Applying the coordinate change $(x, y) \rightarrow(x-y, y)$ in $\mathbf{R}$ transforms $\mathbf{Q}_{1}$ into $\mathbf{Q}_{1}=(x)$ and $f$ into $f=x^{2}-2 x y+y^{5}$. The strict transform in this chart is $f^{\prime}=x^{2}-2 x+y^{3}$ which is smooth at 0 if the characteristic is different 2. For characteristic equal 2 we have $f=(x+y)^{2}+y^{5}$ in the original coordinates and the multiplicity of the strict transform remains 2 at the point $\mathbf{Q}_{1}$. Exercise: Explain how this phenomenon fits into the preceding argument for the maximal slope (cf. [A 2]).

Example 6. Consider the isolated surface singularity $f=x^{2}+y^{3}+y z^{3}$ with the origin as the only permissible center. In the chart $\mathbf{Q}=(x, y)$ we get $f^{\prime}=x^{2}+y^{3} z+y z^{2}$. One monomial has decreased, the other increased. Exercise: Prove that something has improved. Problem: Express the improvement without using coordinates. You may consult [H 3] or [Ha 3, Ha 4] for a detailed discussion. 
For fixed coordinates in $\mathbf{R}$, the multiplicity drops under monomial blowup of type $j \in J$ if and only if the variable $x_{j}$ occurs in the initial form of $f$ (= homogeneous polynomial of lowest degree of $f$ ) defining the tangent cone of the variety at the origin. Hence, for arbitrary blowup, it drops in all charts if and only if the tangent cone of $f$ is not a cartesian product with a line transversal to the center. This observation led Hironaka to define the invariant $\tau$ as the minimal number of variables necessary to define the tangent cone of $f$. Abhyankar proceeds similarly, cf. e.g. [A 4, p. 230]. Above we have seen an example (ex. 5) where $\tau$ equals 2 in characteristic $\neq 2$ and 1 in characteristic 2 . If $\tau=n$ then the multiplicity drops at each point of the exceptional divisor, which suggests to apply induction on $n-\tau$. In characteristic zero, constant multiplicity implies that $\tau$ does not decrease [H 1, III. Thm. 3]. In three variables, this holds in any characteristic, and allows Hironaka to prove resolution of excellent surfaces by studying further invariants deduced from the Newton polyhedron of $f[\mathrm{H} 3$, Co 1$]$. In dimension four he gives the following example of decrease of $\tau$, hence increase of $n-\tau$, an example which initiated a different definition of $\tau$ via additive forms in positive characteristic [H 4, Gi 2, Gi 3, Od 2, Od 3].

Example 7. [H 4, Thm. 3, p. 331, Od 2, p. 300] Let $\mathbf{K}$ be an imperfect field of characteristic 2 admitting elements $a$ and $b$ such that $\mathbf{K}^{2}(a, b)$ has degree 4 over $\mathbf{K}^{2}$. Let $f=x^{2}+a y^{2}+b z^{2}+a b w^{2}+$ (higher order terms). Then $\tau=4$. Blow up the maximal ideal $\mathbf{M}$. The ideal $\mathbf{Q}^{\prime}=$ $\left(x+y z, y^{2}+b, z^{2}+a\right)$ is maximal in the Rees algebra $\mathbf{S}$ and has residue field $\neq \mathbf{K}$. Then up to terms of degree $\geq 3$ the strict transform at $\mathbf{Q}^{\prime}$ equals $f^{\prime}=x^{2}+a y^{2}+b z^{2}+a b=(x+y z)^{2}-\left(y^{2}+b\right)\left(z^{2}+a\right)$ and hence $\tau^{\prime}=3$. This type of phenomenon can of course be avoided by working with algebraically closed ground fields.

(3) Equimultiple locus. One important ingredient in resolution arguments is induction on the number of variables, i.e., on the local embedding dimension. As its definition involves derivatives, it is natural to expect complications in positive characteristic. This happens in particular in the theory of maximal contact. The basic observation in this theory is the simple fact that the equimultiple locus $S$ of a hypersurface singularity in $\mathbf{A}^{n}$ has local embedding dimension less than $n$, provided the characteristic is zero. For given $f$, let $\mathbf{J}$ be the ideal of $\mathbf{R}$ generated by all partial derivatives of $f$ up to degree less than the multiplicity of $f$. Its zero locus is $S$. By definition of the multiplicity of $f$, one of the derivatives appearing among the generators of $\mathbf{J}$ must have order 1 and therefore defines a smooth hypersurface. As $S$ is contained in it, the assertion follows. 
The theory of maximal contact and of idealistic presentations allows, by induction, to resolve first the singularities of $S$. Once $S$ has only normal crossing singularities its components can be chosen as centers of further blowups in order to improve the variety itself. In general, the equimultiple locus of a hypersurface is not a hypersurface. This forces Hironaka to use resolution of non hypersurfaces of lower embedding dimension to resolve hypersurfaces [H 5]. Bierstone and Milman have modified the argument as to get a proof for hypersurfaces in zero characteristic which remains inside this class [BM 1].

The reasoning on the embedding dimension breaks completely down in positive characteristic.

Example 8. Let $\mathbf{K}$ be a field of characteristic 2. Consider $f=x^{2}+$ $y z^{3}+z w^{3}+y^{7} w$ of order 2 at 0 and embedding dimension 4 . The equimultiple locus $S$ is given by the first order partial derivatives of $f$, say $\mathbf{J}=\left(0, z^{3}+y^{6} w, y z^{2}+w^{3}, z w^{2}+y^{7}\right)$. It has embedding dimension 4 since the monomial curve $C$ parametrized by $t^{32}, t^{7}, t^{19}, t^{15}$ lies in $S$ but cannot be embedded locally at 0 into $\mathbf{A}^{3}$.

Compare this with the induction on the number of variables based on the Weierstrass form of a series and the application of Tschirnhausen transformations [A 1, A 5, BM 2, Co 2, Mo 1, Mo 2, Su]. Again this fails in positive characteristic.

There are attempts by Giraud to extend the concept of maximal contact to positive characteristic [Gi 2, Gi 3], but his theory is not fully applicable. We are not aware of any explicit substitute for the local embedding dimension as induction invariant for arbitrary characteristic.

Instead of the local embedding dimension one could try with the dimension of the equimultiple locus. It turns out that it varies quite arbitrarily under blowing up and hence does not serve as a resolution invariant:

Example 9. Take $f=x^{2}+y^{4}+z^{4}$ with isolated singularity at 0 in zero characteristic. Blow up the origin and consider the chart $\mathbf{Q}=(x, y)$. Then $f^{\prime}=x^{2}+y^{4} z^{2}+z^{2}=x^{2}+\left(z \sqrt{y^{4}+1}\right)^{2}$. This singularity is locally isomorphic to the cartesian product of the plane curve $x^{2}+z^{2}$ with a line which forms its equimultiple locus.

For a detailed study of equimultiple curves and their behaviour under blowing up, see [Z 1, Z 6]. Zariski shows that for surfaces the equimultiple locus of the blown up variety can at most consist of the strict transform of the old equimultiple locus plus possibly some new but smooth equimultiple curve. This allows to reduce always by point blowups to a normal crossing equimultiple locus. This is false for threefolds: 
Example 10. The singular and equimultiple locus may become worse under blowing up. Consider the hypersurface $f=x^{2}+y^{3}+z^{2} w+w^{4}$ in characteristic zero. Its Jacobian ideal is $j(f)=\left(x, y^{2}, z w, z^{2}+4 w^{3}\right)$. Hence the singularity at 0 is isolated of multiplicity 2. Blow up the origin and look at the chart corresponding to $\mathbf{Q}=(x, y, z)$. The strict transform of $f$ is given by $f^{\prime}=x^{2}+y^{3} w+z^{2} w+w^{2}$. It has Jacobian ideal $j\left(f^{\prime}\right)=\left(x, y^{2} w, z w, y^{3}+z^{2}+2 w\right)$. The singular locus of $X^{\prime}$ is the singular curve $y^{3}+z^{2}=0$ inside the plane $x=w=0$. As the multiplicity is 2, it coincides with the two-fold locus of $X^{\prime}$.

Example 11. The number of strata appearing in the equimultiple stratification of $X$ at 0 may increase. Consider the hypersurface $f=x^{3}+$ $y^{4}+y z^{2} w+z^{4} w+w^{6}$ in zero characteristic with Jacobian ideal $j(f)=$ $\left(x^{2}, 4 y^{3}+z^{2} w,\left(y+2 z^{2}\right) z w, y z^{2}+z^{4}+6 w^{5}\right)$. Any singular point close to 0 must satisfy $x=z=w=0$ and then $y=0$ follows immediately. So the singularity at 0 is isolated. Blow up the origin and look at the chart corresponding to $\mathbf{Q}=(x, y, z)$. The strict transform of $f$ is given by $f^{\prime}=x^{3}+y^{4} w+y z^{2} w+z^{4} w^{2}+w^{3}$ and has Jacobian ideal $j\left(f^{\prime}\right)=\left(x^{2},\left(4 y^{3}+z^{2}\right) w,\left(y+2 z^{2} w\right) z w, y^{4}+y z^{2}+2 z^{4} w+3 w^{2}\right)$. A short computation shows that the singular locus of $X^{\prime}$ near 0 is contained in the plane $x=w=0$. It then follows that it equals the curve $y\left(y^{3}+z^{2}\right)=0$. Hence the two-fold locus of $X^{\prime}$ is a reducible plane curve with one smooth and one singular component. The three-fold locus is again the origin.

(4) Hilbert-Samuel function. Hironaka's original generalization of the multiplicity to the non hypersurface case is an increasing sequence $\nu^{*}$ of integers defined as follows: given an ideal $\mathbf{I}$ in $\mathbf{R}$ consider a generator system $f_{1}, \ldots, f_{m}$ of $\mathbf{I}$ subject to the following condition: the initial forms of the $f_{i}$ are a minimal generator system of the initial forms ideal of $\mathbf{I}$ (= ideal generated by all initial forms of elements of $\mathbf{I}$ ) and the $f_{i}$ are numbered by increasing multiplicity. Then $\nu^{*}$ is the sequence $\nu_{i}=o\left(f_{i}\right)$ of multiplicities, completed to an infinite sequence by adding infinity. Two sequences are compared pairwise lexicographically. He called such generators a standard basis of $\mathbf{I}$, distinct to nowadays use of the term, where standard bases are defined w.r.t. monomial orders on $\mathbf{N}^{n}$ and referring to initial monomials instead of initial forms. Permissibility of the center (= normal flatness of the variety along it) can be expressed by the equimultiplicity of each element of the standard basis along the center [H 1, II. Thm. 2]. This description allows the explicit construction of the strict transform of the ideal via the strict transforms of its standard basis. Hironaka showed that $\nu^{*}$ does not increase under permissible blowing up. Later on, $\nu^{*}$ was commonly replaced by the Hilbert-Samuel function of the variety, for which Bennett had proven semicontinuity under blowing 
up and localization in arbitrary characteristic [Bn, Si]. Hironaka himself observes that $\nu^{*}$ does not behave well under localization:

Example 12. [H 1, remark p. 220] Let $\mathbf{K}$ be an arbitrary field, and let $\mathbf{I}=\left(x y+\left(x+z^{2}\right) w, x(x+y)+(y+z)^{2} w,(x+z)^{2}(x+y)-(y+z)^{2} y\right)$ in $\mathbf{R}=$ $\mathbf{K}[[x, y, z, w]]$. It has $\nu^{*}=(2,2,3, \infty, \ldots)$. Localizing in $(x, y, z)$ gives $\mathbf{I}_{l o c}=\left(x y+(x+z)^{2} w, x(x+y)+(y+z)^{2} w\right)$ with $\nu_{l o c}^{*}=(2,2, \infty, \ldots)>\nu^{*}$.

Any choice of monomial order allows to refine $\nu^{*}$ by taking instead of initial forms of $\mathbf{I}$ initial monomials w.r.t. to this order, e.g. the graded lexicographic order. The resulting ideal, the initial ideal w.r.t. the order, is a monomial ideal and hence a purely combinatorial object. In contrast to $\nu^{*}$ it depends on the choice of coordinates. It can be made coordinate free by considering the generic initial ideal, i.e., the initial ideal which occurs for a generic choice of coordinates. Genericity makes only sense for infinite ground fields. More conceptually, the set of monomial ideals can be totally ordered by comparing their vertices lexicographically w.r.t. the given monomial order, and then the generic initial ideal coincides with the minimal initial ideal, where the minimum is taken over all choices of coordinates [Ha 1]. This follows from the upper semicontinuity of initial ideals in deformations [BM 4].

If the order is compatible with total degre the initial ideal determines $\nu^{*}$ and the Hilbert-Samuel function, but not conversely, as the induced stratification is in general strictly finer than the Hilbert-Samuel stratification:

Example 13. [Ga, p. 567] Take $\mathbf{I}=\left(x^{2}, x y, x z+y^{2} w, y^{3}\right)$. Its initial ideal w.r.t. the graded lexicographic order with $x<y<z<w$ is in $\mathbf{I}=\left(x^{2}, x y, x z, y^{3}\right)$ and is already the generic initial ideal. The initial ideal stratum is the origin, whereas the Hilbert-Samuel stratum coincides with the $w$-axis. To see this, it suffices to consider $\mathbf{I}_{t}=\left(x^{2}, x y, x z+\right.$ $\left.y^{2}(w-t), y^{3}\right)$ with $t \in \mathbf{K}$ so that in $\mathbf{I}_{t}=\left(x^{2}, x y, y^{2}\right)$. The defect of the initial ideal stratification is the following: the origin is the only permissible center w.r.t. in $\mathbf{I}$, but blowing it up the strict transform $\mathbf{I}^{\prime}$ at the point $\mathbf{Q}=(x, y, z)$ equals $\mathbf{I}$. Hence the stratification is to fine to describe suitable centers.

This shows that initial ideals might be used to define part of the resolution invariant $i$ but they are not suited to define the centers. Actually, the generic or minimal initial ideal still does not contain sufficient information as to measure the improvement of a singularity under blowup: for hypersurfaces, and having a graded monomial order, it just gives the multiplicity. This is due to the fact that the Newton polyhedron degenerates completely in generic coordinates to a polyhedron with one 
compact face. The interesting information appears in very specific coordinates when the vertices of the Newton polyhedron are as remote from the origin as possible, see the discussion below.

(5) Automorphisms. Invariants as the slope, $\tau$ or the initial ideal are examples of a frequent construction of invariants: Choose coordinates $x$ in $\mathbf{R}$, develop $f$ or the generators of the defining ideal into its power series expansion and extract some numerical datum. It will depend on the coordinates. To make it independent take e.g. its minimal or maximal value over all choices of coordinates. This gives an invariant of the singularity. In order to study its behaviour under blowup one proceeds as follows [Ha 1].

Let us restrict to hypersurfaces for simplicity. Let $q_{x} f$ be a numerical datum associated to the power series expansion of $f$ in the coordinates $x$, for which the minimum $q_{\min } f=\min _{x}\left\{q_{x} f\right\}$ over all coordinate choices exists. If the blowup is monomial in the coordinates $x$ and if $y$ denote the induced coordinates in $\mathbf{R}^{\prime}$ the change from $q_{x} f$ to $q_{y} f^{\prime}$ is computed by substituting in the expansion of $f$ the variables $x$ by the corresponding monomials in $y$ according to the formula for *. Assume that we can show in this way that $q_{x} f>q_{y} f^{\prime}$ for monomial blowups. Now, coordinates for which the blowup is monomial need not realize the minimal value of $q$. Assume, however, that there exists a coordinate change $x \rightarrow \tilde{x}$ in $\mathbf{R}$ which realizes the minimum and such that the blowup stays monomial w.r.t. $\tilde{x}$. We then get

$$
q_{\min } f=q_{\tilde{x}} f>q_{\tilde{y}} f^{\prime} \geq q_{\min } f^{\prime}
$$

where $\tilde{y}$ denote the coordinates in $\mathbf{R}^{\prime}$ induced from $\tilde{x}$. This shows that the minimum $q_{\min } f$ has dropped. Observe that it was not necessary to realize the minimum in $\mathbf{R}^{\prime}$. We only used that $q_{x} f>q_{y} f^{\prime}$ under monomial blowup and that there is a coordinate change in $\mathbf{R}$ realizing the minimum and preserving monomiality. It turns out that if $q_{x} f$ is defined through a monomial order as a certain initial monomial of $f$ the minimizing coordinate changes can be chosen from a product $S \mathbf{U}$ inside the automorphism group of $\mathbf{R}$, where $S$ is the permutation group on the coordinates and where $\mathbf{U}$ is a generalized 'unipotent' subgroup [Ha 2]. This relies on the Gauss-Bruhat decomposition of Aut $\mathbf{R}$. The explicit description of these subgroups allows to determine the cases where it is possible to choose a minimizing coordinate change in $\mathbf{R}$ such that the monomiality of the blowup is preserved.

For maxima, the argument is upside down. Assume again that we have $q_{x} f>q_{y} f^{\prime}$ for monomial blowups. Now suppose that there exists a maximizing coordinate change $y \rightarrow \tilde{y}$ in $\mathbf{R}^{\prime}$ which is induced from a 
coordinate change in $\mathbf{R}$ and such that the monomiality of the blowup is preserved. Then

$$
q_{\max } f \geq q_{\tilde{x}} f>q_{\tilde{y}} f^{\prime}=q_{\max } f^{\prime}
$$

where $\tilde{x}$ denote the coordinates in $\mathbf{R}$ obtained from $x$ by the coordinate change. The maximum $q_{\max } f$ has dropped. It was not necessary to realize it in $\mathbf{R}$.

Note that if $q_{x} f$ belongs to a well ordered set, its minimum always exist, whereas the maximum need not, even in case the set $\left\{q_{x} f\right\}$ is bounded from above. Nevertheless, if $q_{x} f$ is upper semicontinuous w.r.t. $x$ the maximum, if it exists, is in general much more sensitive to improvements of the singularity because it corresponds to very special choices of coordinates. If $q_{x} \mathbf{I}$ is defined as the initial ideal of $\mathbf{I}$ w.r.t. a given monomial order the maximum exists as is shown by a double application of Artin's Approximation Theorem and using the standard basis criterion of Becker-Buchberger [Ha 1].

To realize maxima in $\mathbf{R}^{\prime}$ is more difficult then to realize minima in $\mathbf{R}$ when required to preserve the monomial situation. One of the reasons is that for automorphisms of $\mathbf{R}^{\prime}$ to be induced from automorphisms of $\mathbf{R}$, it is necessary but not sufficient that they fix the exceptional divisor $D$ of the blowup:

Example 14. Let $\mathbf{R}^{\prime}$ be the local ring obtained from $\mathbf{R}=\mathbf{K}[[x, y, z]]$ by blowing up the maximal ideal and looking at the chart $\mathbf{Q}=(x, y)$. Then automorphisms $g$ of $\mathbf{R}^{\prime}$ of form $g(x, y, z)=\left(x+y^{2}, y, z\right)$ fix the exceptional divisor $z=0$ but are not induced from automorphisms in $\mathbf{R}$ preserving the monomial situation.

Maximal initial ideals seem to be very appropriate to be used as resolution invariants. They appear implicitly and in modified form for hypersurfaces in various papers of Abhyankar, Hironaka, Moh and others, and each time their existence is proven by hand. The technique of [Ha 1] provides a simultaneous proof of these results. Yet there is another difficulty to apply initial ideals successfully. Under monomial blowup, the expansion of $f$ transforms under the map * into the expansion of $f^{\prime}$, but the initial monomial of $f^{\prime}$ need not be the transform of the initial monomial of $f$. Hence direct comparison is sometimes impossible.

Example 15. Let $f=x^{5}+x^{4} y^{2}+y^{4} z^{5}$. Consider the monomial order on $\mathbf{N}^{3}$ given by $\alpha<\beta$ if $\left(\alpha_{1}+\alpha_{3}, \alpha_{2}, \alpha_{1}\right)<\left(\beta_{1}+\beta_{3}, \beta_{2}, \beta_{1}\right)$ lexicographically. Then $x^{4} y^{2}$ is the initial monomial of $f$. Blowup the origin and look at the chart given by $\mathbf{Q}=(x, y)$. Then $f^{\prime}=x^{5}+x^{4} y^{2} z+y^{4} z^{4}$ and its initial monomial is $y^{4} z^{4}$. 
Monomial orders are hence in general not compatible in all charts with monomial blowup. It might be possible to overcome this obstruction by considering a refinement of the notion of monomial orders, the so called monomial rotation orders introduced in $[\mathrm{Ha} 6]$. These are defined by rotating a hyperplane in $\mathrm{R}_{+}^{n}$ around a fixed vertex and taking as initial monomials those whose exponents are touched first by this hyperplane. One recovers all vertices which are adjacent to the selected one, cf. [Ha $3]$ and the notion of critical tropism [LT].

(6) Relative Multiplicity. This is an invariant suggested by Abhyankar and Hironaka. It is used by Spivakovsky in his solution to Hironaka's polyhedral game and by Moh and Cossart for resolution of threefolds [Sp 2, Co 2, Mo 1, Mo 2]. It also appears in the constructive resolutions of Bierstone-Milman and Villamayor [BM 1, V 1]. We describe it in the simplest possible context. Consider a power series $f$ in three variables of form $f(x, y, z)=x^{o}+g(y, z)$ with $g$ of order $>o$. Factor from $g$ the largest monomial in $y$ and $z$, say $g(y, z)=y^{i} z^{k} h(y, z)$. The relative multiplicity of $f$ w.r.t. the given coordinates is defined as the order of $h$ w.r.t. $y$ and $z$. It depends on $x, y, z$ and will be denoted by $r_{x y z} f$. In order to get a coordinate independent invariant consider all coordinates $x, y, z$ in which $f$ has the form $f=x^{o}+g(y, z)$. Among these, take those $x$ for which the Newton polygon of $g$ is minimal settheoretically. For fixed $y$ and $z$, it can be checked that the minimal Newton polygon is unique. Hence it only depends on $y$ and $z$. Now vary $y$ and $z$. In the presence of components of the exceptional divisor, $y$ and $z$ are subject to define them by $y=0$ and/or $z=0$; else there is no condition. Next, choose $y$ and $z$ such that the monomial factored from $g$ has highest possible degree $i+k$. In all such coordinates the order of $h$ is the same and called the relative multiplicity $r(f)$ of $f$ (in the literature, this number is usually divided by $o$ and called the weighted order of $f$.)

The definition is relatively involved and not as conceptual as one would wish. Moreover, in positive characteristic, $r$ may increase under blowing up. Also it does not behave well under deformations:

Example 16. [Mo 2, ex. 3.2] Let $f=x^{p}+y^{2} z^{3 p-2}+y z^{3 p+1}+z^{3 p+2}=$ $x^{p}+z^{3 p-2}\left(y^{2}+y z^{3}+z^{4}\right)$ where $p$ is the characteristic of $\mathbf{K}$. Here $r_{x y z} f=2 \neq r(f)$. Apply the coordinate change $\varphi=\left(x-z^{3}, y-z, z\right)$ and get $f_{1}=\varphi^{*} f=x^{p}+y z^{3 p-2}\left(y-2 z+z^{3}\right)$. Then $r_{x y z} f_{1}=r\left(f_{1}\right)=r(f)=1$. Blow up the origin and consider the point corresponding to $\mathbf{Q}=(x, y)$. The exceptional divisor $D$ is given by $z=0$ and the strict transform of $f$ equals $f^{\prime}=x^{p}+y^{2} z^{2 p}+y z^{2 p+2}+z^{2 p+2}=x^{p}+z^{2 p}\left(y^{2}+y z^{2}+z^{2}\right)$. We have $r_{x y z} f^{\prime}=r\left(f^{\prime}\right)=2>r(f)$.

Example 17. Let $f=x^{p}+y^{m} z^{2 p}\left(z^{p}+y^{p+1}+z^{p+1}\right)$ with $r(f)=p$. 
Consider the deformation $f_{t}=x^{p}+(y-t)^{m} z^{2 p}\left(z^{p}+y^{p+1}+z^{p+1}\right)$. Applying the automorphism $\varphi=\left(x-t^{m / p} z^{3}, y, z\right)$ gives $\varphi^{*} f_{t}=x^{p}+$ $z^{2 p}\left(y z^{p} u_{t}+y^{p+1}+z^{p+1}\right)$ with some unity $u_{t}$ of $\mathbf{R}$ and $r\left(f_{t}\right)=p+1$ if $t \neq 0$. Hence the relative multiplicity is not upper semicontinuous under deformation.

\section{References}

[A 1] Abhyankar, S.: Local uniformization of algebraic surfaces over ground fields of characteristic $p \neq 0$. Ann. Math. 63 (1956), 491-526. [One of the first contributions to the global resolution problem in positive characteristic.]

[A 2] Abhyankar, S.: Desingularization of plane curves. In: Summer Institute on Algebraic Geometry. Arcata 1981, Proc. Symp. Pure Appl. Math. 40 Amer. Math. Soc. [Discusses invariants as the maximal slope for plane curves and explains problems which are special for positive characteristic. Describes Tschirnhausen transformations.]

[A 3] Abhyankar, S.: Current status of the resolution problem. Summer Institute on Algebraic Geometry 1964. Proc. Amer. Math. Soc. [Surveys the situation before and around Hironaka's Annals paper.]

[A 4] Abhyankar, S.: Algebraic Geometry for Scientists and Engineers. Math. Surveys and Monographs 35. Amer. Math. Soc. 1990. [Provides a description of the state of the art in resolution of singularities and related problems (p. 253).]

[A 5] Abhyankar, S.: Analytic desingularization in characteristic zero. Preprint 1996. [Local resolution of hypersurfaces, cf. with [BM 2].]

[Al] Albanese, G.: Transformazione birazionale di una superficie algebrica qualunque in un altra priva di punti multipli. Rend. Circ. Mat. Palermo 48 (1924).

[AJ] Abramovich, D., de Jong, J.: Smoothness, semi-stability and toroidal geometry. Preprint 1996. [Short proof a weak version of Hironaka's Theorem in characteristic zero, cf. with [BP].]

[Ar] Artin, M.: Lipman's proof of resolution of singularities. In: Arithmetic Geometry (eds. G. Cornell, J. H. Silverman). [Surveys the paper [Lp 2] on resolution of two dimensional schemes.]

[Bb] Brandenberg, M.: Aufblasungen affiner Varietäten. Diplomarbeit Zürich 1992. [Detailed introduction to blowup.] 
[Bd] Brodmann, M.: Computerbilder von Aufblasungen. El. Math. 50 (1995), 149-163.

[Be] Berthelot, P.: Altérations de variétés algébriques (d'après A.J. de Jong). Sém. Bourbaki, exp. 815 (1995/96). [Reviews the proof and gives various applications of de Jong's result.]

[BK] Brieskorn, E., Knörrer, H.: Ebene algebraische Kurven. Birkhäuser 1981. [Discusses in detail slopes of Newton polygon and maximal contact.]

[BM 1] Bierstone, E., Milman, P.: Canonical desingularization in characteristic zero by blowing up the maximum strata of a local invariant. To appear in Invent. Math. [Systematic presentation of resolution invariants and treatment of non hypersurface case.]

[BM 2] Bierstone, E., Milman, P.: Uniformization of analytic spaces. J. Amer. Math. Soc. 2 (1989), 801-836. [Establishes a local version of resolution in the analytic category, cf. with [A 5].]

[BM 3] Bierstone, E., Milman, P.: A simple constructive proof of canonical resolution of singularities. In: Effective Methods in Algebraic Geometry (eds. T. Mora, C. Traverso). Progress in Math. 94, Birkhäuser 1991, 11-30.

[BM 4] Bierstone, E., Milman, P.: Relations among analytic functions I. Ann. Inst. Fourier 37 (1987), 187-239. [Includes study on initial ideals.]

[Bn] Bennett, B.-M.: On the characteristic function of a local ring. Ann. Math. 91 (1970), 25-87. [Proves that the Hilbert-Samuel function does not increase under permissible blowing up in any characteristic and that it is upper-semicontinuous under localization.]

[BP] Bogomolov, F., Pantev, T.: Weak Hironaka Theorem. To appear in Math. Res. Letters. [Short proof a weak version of Hironaka's Theorem in characteristic zero, cf. with [AJ].]

[Br 1] Brieskorn, E.: Über die Auflösung gewisser Singularitäten von holomorphen Abbildungen. Math. Ann. 166 (1966), 76-102.

[Br 2] Brieskorn, E.: Die Auflösung der rationalen Singularitäten holomorpher Abbildungen. Math. Ann. 178 (1968), 255 - 270.

[Ch] Chisini, O.: La risoluzione delle singolarità di una superficie mediante transformazioni birazionali dello spazio. Mem. Accad. Sci. Bologna VII. 8 (1921).

[CGO] Cossart, V., Giraud, J., Orbanz, U.: Resolution of surface singularities. Lecture Notes in Math. vol. 1101, Springer 1984. 
[Compares resolution methods for surfaces by Abhyankar, Hironaka, Jung and Zariski.]

[Co 1] Cossart, V.: Desingularization of embedded excellent surfaces. Tôhoku Math. J. 33 (1981), 25-33. [Fills a gap in [H 3] by extending H.'s argument to non rational points.]

[Co 2] Cossart, V.: Polyèdre caractéristique d'une singularité. Thèse d'Etat, Orsay 1987. [Normal form of functions in three variables under blowup in characteristic $p$.]

[Co 3] Cossart, V.: Désingularisation des surfaces (d'après Zariski). Preprint Ecole Polytechnique 1995.

[CP] Cano, F., Piedra, R.: Characteristic polygon of surface singularities. In: Géométrie algébrique et applications II (eds. J.-M Aroca, T. Sanchez-Giralda, J.-L. Vicente), Proc. of Conference on Singularities, La Rábida 1984. Hermann 1987.

[Ga] Galligo, A.: A propos du Théorème de Préparation de Weierstrass. Springer Lecture Notes in Math. 409 (1973), 543-579.

[Gi 1] Giraud, J.: Etude locale des singularités. Cours de $3^{e}$ Cycle, Orsay 1971/72. [Exposition of basic notions for resolution.]

[Gi 2] Giraud, J.: Sur la théorie du contact maximal. Math. Z. 137 (1974), 285-310. [Discusses definition of $\tau$ in positive characteristic.]

[Gi 3] Giraud, J.: Contact maximal en caractéristique positive. Ann. Scient. E.N.S. 8 (1975), 201-234.

[Gr 1] Grothendieck, A.: Traveaux de Heisouke Hironaka sur la résolution des singularités. Actes Congrès International, Nice 1970. [Short summary, admits that he has not got through the paper completely.]

[Gr 2] Grothendieck, A.: EGA IV. Publ. Math. IHES 24 (1965).

[GT] Goldin, R, Teissier, B.: Resolving singularities of plane analytic branches with one toric morphism. Preprint ENS Paris 1995. [Uses toric modifications to resolve plane curve singularities.]

$[\mathrm{H}$ 1] Hironaka, H.: Resolution of singularities of an algebraic variety over a field of characteristic zero. Ann. of Math. 79 (1964), 109-326. [Comprehensive presentation of many concepts used in resolution arguments, such as blowup, strict and total transform, normal flatness, standard bases. To a large extent very clear and informative, the complicated part being the proper induction argument.] 
[H 2] Hironaka, H.: Characteristic polyhedra of singularities. J. of Math. Kyoto Univ. 7 (1967), 251-293. [Develops coordinate free Newton polygon, no application to blowup.]

[H 3] Hironaka, H.: Desingularization of excellent surfaces. Notes by B. Bennett at the Conference on Algebraic Geometry, Bowdoin 1967. Reprinted in: Cossart, V., Giraud, J., Orbanz, U.: Resolution of surface singularities. Lecture Notes in Math. 1101, Springer 1984. [Explicit study of Newton polyhedron to make multiplicity drop in a finite number of blowups. The argument has to be complemented by [Co 1] for non rational points.]

[H 4] Hironaka, H.: Additive groups associated with points of a projective space. Ann. Math. 92 (1970), 327-334. [Discussion of $\tau$ in positive characteristic.]

[H 5] Hironaka, H.: Idealistic exponents of singularity. In: Algebraic Geometry, the Johns Hopkins Centennial Lectures. Johns Hopkins University Press 1977. [Development of division theorem to study standard bases and their role in resolution arguments. Treats non hypersurfaces through the notion of idealistic presentation.]

[H 6] Hironaka, H.: Schemes etc. In: 5th Nordic Summer School in Mathematics (ed. F. Oort), Oslo 1970, 291-313. [Uses differential operators to investigate $\tau$. Contains various examples.]

[H 7] Hironaka, H.: Desingularization of complex analytic varieties. Actes Congrès Intern. Math., Nice 1970, 627-631.

[Ha 1] Hauser, H.: Maximal and minimal initial ideals in resolution processes. Preprint Innsbruck 1996. [Proves that the minimal and maximal initial ideal over all coordinate choices exists. Short proof of infinitesimal semicontinuity of Hilbert-Samuel function. Studies maximal initial ideals as resolution invariants.]

[Ha 2] Hauser, H.: Gauss-Bruhat decomposition, flag varieties and blowing up. Preprint Innsbruck 1996. [Establishes product decomposition for automorphisms of power series rings. Allows to determine maximizing and minimizing coordinate changes. Applies to construct flag invariants from the Newton polyhedron.]

[Ha 3] Hauser, H.: Triangles, prismas and tetrahedra as resolution invariants. Preprint Innsbruck 1996. [Resolves Hironaka's polyhedral game in three variables. Uses theory of convex polytopes to construct new resolution invariants.]

[Ha 4] Hauser, H.: A critical reading of Hironaka's Bowdoin lecture on desingularization of excellent surface singularities. Preprint 
Innsbruck 1996. [Provides conceptual foundation of [H 3]. Describes advantages and limitations of H.'s approach.]

[Ha 5] Hauser, H.: The concept of blowing up in algebraic geometry. In preparation.

[Ha 6] Hauser, H.: Monomial rotation orders. In preparation.

[HIO] Herrmann, M., Ikeda, S., Orbanz, U.: Equimultiplicity and blowing up. Springer 1988. [Discusses in detail concepts as multiplicity, normal flatness, Hilbert-Samuel function, etc.]

[Hs] Hartshorne, R.: Algebraic Geometry. Springer 1977.

[J] de Jong, A.: Smoothness, semi-stability and alterations. To appear in Publ. Math. IHES. ['The' new approach to resolution of singularities through semi-stable reduction.]

[Ju] Jung, H.: Darstellung der Funktionen eines algebraischen Körpers zweier unabhängiger Veränderlicher $x, y$ in der Umgebung einer Stelle $x=a, y=b$. J. Reine Angew. Math. 133 (1908), 289-314. [Proves local resolution for complex surfaces. His method is reviewed in [Lp 1].]

[K] Kunz, E.: Algebraische Geometrie IV. Vorlesung Regensburg. [Lectures on Hironaka's Annals paper 1964.]

[KKMS] Kempf, G., Knudson, F., Mumford, D., Saint-Donat, B.: Toroidal Embeddings I. Lect. Notes Math. 339 Springer 1973. [Theory of toric modification and resolution.]

[La] Laufer, H.: Normal two-dimensional singularities. Ann. Math. Studies 71. [Explicit resolution of normal surface singularities. Includes examples.]

[LO] Lê, D.T., Oka, M.: On resolution complexity of plane curves. Kodai Math. J. 18 (1995), 1-36. [Describes number of blowups necessary to resolve plane curve singularities.]

[Lp 1] Lipman, J.: Introduction to resolution of singularities. Proceedings Symp. Pure Appl. Math. 29 Amer. Math. Soc. 1975, 187-230. [Surveys various methods of resolution by Jung, Walker, Zariski and Abhyankar.]

[Lp 2] Lipman, J.: Desingularization of 2-dimensional schemes. Ann. Math. 107 (1978), 151-207. [Resolution based on homological methods, duality and differentials.]

[LT] Lejeune, M., Teissier, B.: Contribution à l'étude des singularités du point de vue du polygone de Newton. Thèse d'Etat 1973.

[Lv 1] Levi, B.: Sulla risoluzione delle singolarità puntuali delle superficie algebriche dello spazio ordinario per transformazioni quadratiche. Ann. Mat. Pura Appl. II 26 (1897). 
[Lv 2] Levi, B.: Risoluzione delle singolarità puntuali delle superficie algebriche. Atti Acad. Sci. Torino 33 (1897), 66-86.

[Mo 1] Moh, T.-T.: Canonical uniformization of hypersurface singularities of characteristic zero. Comm. Alg. 20 (1992), 3207-3249.

[Mo 2] Moh, T.-T.: On a Newton polygon approach to the uniformization of singularities of characteristic $p$. In: Algebraic Geometry and Singularities (eds. A. Campillo, L. Narváez). Proc. Conf. on Singularities La Rábida. Birkhäuser 1996. [Includes example that weighted order may increase in positive characteristic.]

[N] Noether, M.: Über einen Satz aus der Theorie der algebraischen Funktionen. Math. Ann. 6 (1873), 351-359.

[NB] Noether, M., Brill, A.: Die Entwicklung der Theorie der algebraischen Funktionen in älterer und neuerer Zeit. Jahresber. Dt. Math. Verein. III (1892/93), 107-566. [Resolution of complex curves. Compares the methods of Kronecker, Noether and Hamburger.]

[Od 1] Oda, T.: Infinitely very near singular points. Adv. Studies Pure Math. 8 (1986), 363-404. [Characteristic free survey on Hilbert-Samuel function and $\nu^{*}$ w.r.t. resolution problems.]

[Od 2] Oda, T.: Hironaka's additive group scheme. In: Number Theory, Algebraic Geometry and Commutative Algebra, in honor of Y. Akizuki, Kinokuniya, Tokyo, 1973, 181-219.

[Od 3] Oda, T.: Hironaka group schemes and resolution of singularities. In: Proc. Conf. on Algebraic Geometry, Tokyo and Kyoto 1982. Lecture Notes in Math. 1016, Springer 1983, 295-312.

[Ok] Oka, M.: Geometry of plane curves via toroidal resolution. In: Algebraic Geometry and Singularities (eds. A. Campillo, L. Narváez). Proc. Conf. on Singularities La Rábida. Birkhäuser 1996.

[OW] Orlik, P., Wagreich, P.: Equivariant resolution of singularities with $\mathbf{C}^{*}$ action. In: Proceedings of the 2 nd Conference on Compact Transformation Groups, Amherst 1971.

[P] Pfeifle, J.: Blowing up non reduced normal crossing ideals. Diplomarbeit, Innsbruck 1996. [Investigates non reduced monomial ideals which give smooth varieties when blown up in some ambient space.]

[SG 1] Sánchez-Giralda, T.: Teoria de singularidades de superficies algebroides sumergidas. Monografias y memorias de Matemática IX, Publ. del Instituto Jorge Juan de Matemáticas. Madrid 1976. 
[SG 2] Sánchez-Giralda, T.: Caractérisation des variétés permises d'une hypersurface algébroide. C.R. Acad. Sci. Paris 285 (1977), 1073-1075. [Determines the cases where the multiplicity does not drop under blowup.]

[Si] Singh, B.: Effect of permissible blowing up on the local Hilbert function. Invent. Math. 26 (1974), 201-212. [Refinement of Bennett's result on the infinitesimal semicontinuity of the Hilbert-Samuel function.]

[Sp 1] Spivakovsky, M.: Resolution of Singularities. Preprint 1995. [Preliminary version. Combines Zariski's valuation theory with Hironaka's approach.]

[Sp 2] Spivakovsky, M.: A solution to Hironaka's Polyhedra Game. In: Arithmetic and Geometry. Papers dedicated to I.R. Shafarevich on the occasion of his sixtieth birthday, vol II (eds.: M. Artin, J. Tate). Birkhäuser 1983, 419-432.

[Sp 3] Spivakovsky, M.: A counterexample to Hironaka's 'hard' polyhedra game. Publ. RIMS, Kyoto University 18 (1983), 10091012 .

[Sp 4] Spivakovsky, M.: A counterexample to the theorem of Beppo Levi in three dimensions. Invent. Math. 96 (1989), 181-183.

[Su] Sussmann, H.: Real-analytic desingularization and subanalytic sets: an elementary approach. Trans. Amer. Math. Soc. 317 (1990), 417-461.

[V 1] Villamayor, O.: Constructiveness of Hironaka's resolution. Ann. Sci. Ec. Norm. Sup. Paris 22 (1989), 1-32. [Develops a constructive resolution algorithm and shows that local constructions patch to global ones.]

[V 2] Villamayor, O.: Patching local uniformizations. Ann. Sci. Ec. Norm. Sup. Paris 25 (1992 ), 629-677. [Proves equivariant resolution w.r.t. group actions.]

[V 3] Villamayor, O.: An introduction to the algorithm of resolution. In: Algebraic Geometry and Singularities (eds. A. Campillo, L. Narváez). Proc. Conf. on Singularities La Rábida. Birkhäuser 1996.

[W] Walker, R.J.: Reduction of singularities of an algebraic surface. Ann. Math. 36 (1935), 336-365. [Resolution of surface singularities over $\mathbf{C}$.]

[Y] Youssin, B.: Newton polyhedra without coordinates. Mem. AMS 433 (1990), 1-74, 75-99. 
[Z 1] Zariski, O.: Reduction of singularities of algebraic three dimensional varieties. Ann. Math. 45 (1944), 472-542. [Resolution of three-fold singularities over a field of characteristic 0.$]$

[Z 2] Zariski, O.: Local uniformization theorem on algebraic varieties. Ann. Math. 41 (1940), 852-896.

[Z 3] Zariski, O.: The reduction of singularities of an algebraic surface. Ann. Math. 40 (1939), 639-689. [Resolution of surface singularities over a field of characteristic 0 .]

[Z 4] Zariski, O.: A simplified proof for resolution of singularities of an algebraic surface. Ann. Math. 43 (1942), 583-593.

[Z 5] Zariski, O.: Exceptional singularities of an algebraic surface and their reduction. Atti Accad. Naz. Lincei Rend. Cl. Sci. Fis. Mat. Natur., serie VIII, 43 (1967), 135-196.

[Z 6] Zariski, O.: A new proof of the total embedded resolution theorem for algebraic surfaces. Amer. J. Math. 100 (1978), 411-442.

Mathematisches Institut

Universität Innsbruck

A-6020 Austria.

herwig.hauser@uibk.ac.at

December 1996 